\title{
EVALUATION OF EFFECTIVENESS OF PATCH BASED IMAGE CLASSIFICATION TECHNIQUE USING HIGH RESOLUTION WORLDVIEW-2 IMAGE
}

\author{
Muhammed Yusuf OZTURK ${ }^{1}$, Ismail COLKESEN ${ }^{1}$ \\ ${ }^{1}$ Gebze Technical University, Department of Geomatics Engineering, Gebze-Kocaeli, Turkey \\ (m.ozturk2020, icolkesen)@gtu.edu.tr
}

KEY WORDS: Remote Sensing, LULC, Pixel-Based, Patch-Based, XGBoost, CatBoost

\begin{abstract}
:
The aim of the current study was to evaluate the performance of patch-based classification technique in land use/land cover classification and to investigate the effect of patch size in thematic map accuracy. To reach desired goal, recently proposed ensemble learning classifiers (i.e., XGBoost and CatBoost) were utilized to classify produced image patches obtained from high-resolution WorldView-2 (WV-2) satellite image. . In order to analyse the effect of varying patch size on classification accuracy, three different window sizes (i.e., $3 \times 3,7 \times 7$ and $11 \times 11$ ) were applied to WV-2 imagery for extracting image patches. Constructed image patches were classified using XGBoost and CatBoost ensemble learning classifiers and thematic maps were constructed for varying patch sizes. Results showed that while XGBoost and CatBoost showed similar classification performances for varying patch size and the estimated highest overall accuracy were $\% 68, \% 82$ and $\% 92$ for $11 \times 11,7 \times 7$ and $11 \times 11$ patch sizes, respectively. These findings confirmed that defining class boundaries on the high-resolution image using smaller patches increases the accuracy of thematic maps. In addition, results of patch-based classification were compared the results of LULC maps produced by same classifiers using pixel-based classification method. Overall accuracy of pixel-by-pixel classification of WV-2 image reached to about \%94. Furthermore, CatBoost showed superior classification performance in all time compared to XGBoost. All in all, pixel-based CatBoost was found to be more successful in LULC mapping of fine resolution image.
\end{abstract}

\section{INTRODUCTION}

Producing and visualization of accurate, reliable and actual spatio-temporal information about the Earth's surface plays an important role in planning, monitoring and management of intelligent cities. Land use/land cover (LULC) mapping of surface objects by performing various terrestrial measurements and statistical calculations is one of the ways for collecting information (Luo et al., 2021). In recent years, parallel to the technological advances, there has been increasing interest in the use of remote sensing technologies due to its ability for monitoring large areas at different spectral, spatial and temporal resolution with low cost. Thanks to the recent advances in sensor technology, it is possible to map the spatial distribution of LULC classes with higher accuracy using multiple spectral bands at high spatial resolution (Omer et al., 2015; Colkesen, Ertekin, 2020). Although the quality and resolution of remotely sensed imagery increases, accuracy of LULC maps produced through image classification process can be influenced by the several factors, such as selection of suitable classification technique and the use of robust prediction algorithm.

There are different classification techniques have been applied to multi-spectral, hyperspectral, UAV or Radar images to determinate LULC classes of lands in many studies (Dingle Robertson, King, 2011; Sharma et al., 2017; Zheng et al., 2017; Xu et al.,, 2018; Ozturk and Colkesen, 2021). Traditional pixelbased classification is one of the well-known classification techniques in the literature. The main idea behind the pixelbased classification is to assign each image pixel to certain the LULC classes using their spectral values. Thanks to the high spatial and spectral resolution images provided by recent sensors mounted on different platforms such as drones and satellites, acquisition of more detailed information about the Earth's surface has become possible. Due to the high spatial resolution, the performance of the pixel-based technique may be insufficient for the classification of a large number of pixels with spectrally similar properties. (Colkesen, Kavzoglu, 2017). To overcome these problems and to produce more accurate thematic maps, object-based image classification techniques have been suggested in the literature. The technique mainly relies on image segmentation process grouping spectrally similar pixels to construct image objects. The created image objects then classified considering their geometrical, textural, contextual or statistical properties (Lizarazo, Elsner, 2009). In the last decade, researchers shifted their focus to patch-based classification forming basis of deep learning applications due to their capacity to extract spatial information from image patches using relationship between neighbouring pixels and its ability to reduce noise (Zhu et al., 2017; Yang et al., 2020). Selection of optimal patch size for producing LULC maps is one the key parameters effected the success of patch-based classification. Many studies have been conducted to evaluate the impact of patch size on land cover mapping. For example, the comparative study on the selection of the appropriate vegetation patch size on the accurate mapping was carried out by Lechner et al. (2009). Dumitru et al. (2016) assessed the effect of patch size on generating of thematic maps for urbanized areas using SAR images. On the other hand, the use of a robust and effective classification algorithm for labelling LULC classes is another remarkable factor affecting accuracy of results as well as choosing the appropriate classification technique. The superior performance of ensemble learning algorithms consisting of multiple classifiers in separating class boundaries has been used in many studies (Immitzer et al., 2012; Islam et al., 2020; Bui et al., 2021). XGBoost and CatBoost, one of the recently introduced advanced ensemble classifiers, have been widely used in land cover classification of remotely sensed datasets using pixel-based and object-based process (Gašparović and Dobrinić, 2020; Hamedianfar et al., 2020; Lebedev et al., 2020; Rumora et al., 2020). However, there are few studies about adopting of these algorithms to patch-based classification for producing LULC mapping in the literature ( $\mathrm{Pal}$ et al., 2020; Samat et al., 2020)

The aim of this study is to adopt the patch-based classification and investigate optimal patch size for accurate LULC mapping of WV-2 image. In addition, traditional pixel-based classification was also applied to $\mathrm{WV}-2$ image to find appropriate classification technique for LULC mapping. 
XGBoost and CatBoost were used to classify remotely sensed image and results were evaluated with overall accuracy, kappa coefficient and F-score measures.

\section{STUDY AREA AND DATASET}

The study area covers approximately 220 ha of Bayramoglu Peninsula located in the west of Darica district of Kocaeli province, Turkey. The Bayramoglu Peninsula is located in Darica district of Kocaeli Province and it situated on the coast of Marmara Sea and the south-east of the Istanbul (Figure 1). The study area stands out with sandy beaches and recreation areas, single family houses having private pools and gardens. Generally, eight main LULC classes were determined for Bayramoglu Peninsula: forest including deciduous and coniferous trees, gray surface including roads and gray roofs, pasture, shadow, soil, tile roof, water including sea and pool and white surface including pool tiles and white roofs.

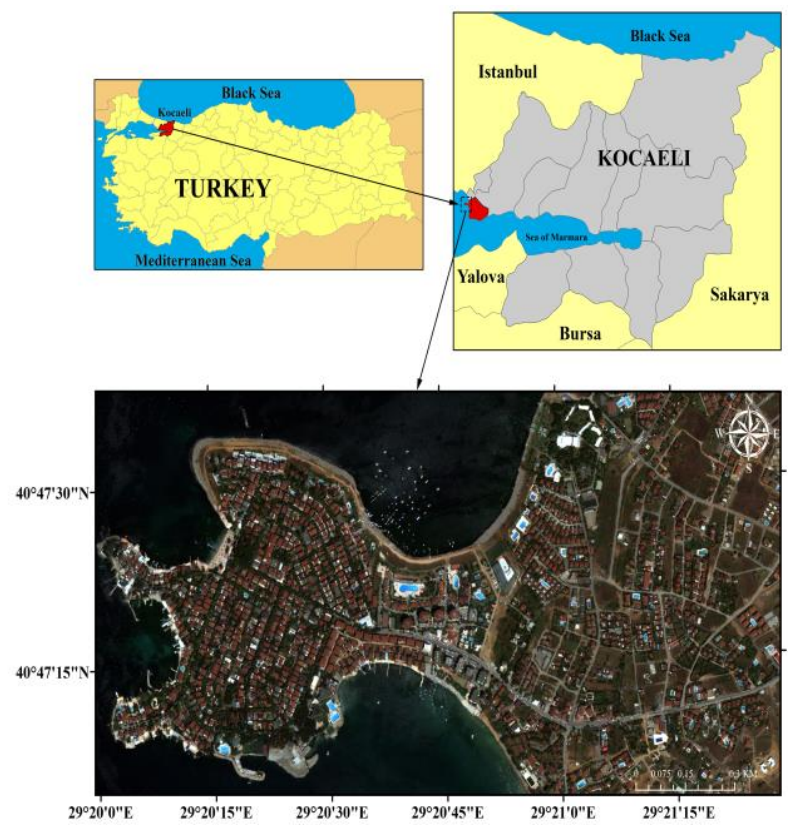

Figure 1. The location of study area

To produce LULC thematic maps of study area by pixel-based and patch-based image classification process, radiometrically and geometrically corrected 16-bit level 2 WorldView-2 (WV2) satellite image acquired on 7 July 2013 was utilized as main data source. The image consists of 8 spectral bands at $2 \mathrm{~m}$ spatial resolution in the central wavelength of $0.427 \mu \mathrm{m}$ (coastal blue), $0.478 \mu \mathrm{m}$ (blue), $0.546 \mu \mathrm{m}$ (green), $0.608 \mu \mathrm{m}$ (yellow), $0.659 \mu \mathrm{m}$ (red), $0.724 \mu \mathrm{m}$ (red edge), $0.831 \mu \mathrm{m}$ (NIR-1) and $0.908 \mu \mathrm{m}$ (NIR-2). It also provides a panchromatic band at 0.5 $\mathrm{m}$ spatial resolution in the spectral range of $0.46-0.80 \mu \mathrm{m}$. To improve the spatial resolution of WV-2 image and enhance interpretability of surface objects, multi-spectral bands were fused with panchromatic band of WV-2 by applying GramSchmidt pan-sharpening algorithm before the image classification process and 8 spectral bands with a spatial resolution of $0.5 \mathrm{~m}$ were obtained.

\section{METEDOLOGY}

In this paper, a comparative study was conducted to analyse the accuracy of classification of WV-2 image by XGBoost and CatBoost using patch-based and pixel-based image analysis methods. In addition, effects of different window sizes (i.e., patch sizes) on estimated classification accuracy were also evaluated. The main processing steps of both classification techniques, description of classifiers and accuracy assessment procedure used for evaluating the accuracies of thematic maps were given in detail in the following subsections.

\subsection{Image Classification}

Different classification techniques, namely pixel-based, subpixel-based, object-based and patch-based have been suggested and applied in remote sensing to classify land cover objects more accurately, efficiently and faster. In recent years, there has been remarkable interest to patch-based classification due to its simple but robust performance (Samat et al., 2020).

In this study, to conduct patch-based classification task two main processing steps were followed. First, square vectors of predefined sizes (i.e., image patches) of high-resolution WV-2 image were produced. Spectral values of each band were extracted using the spatial information of these polygons and added to relevant vectors as attributes with flattening process. Finally, WV-2 raster image with 8 spectral bands was converted into vectorized image using $11 \times 11,7 \times 7$ and $3 \times 3$ window size as shown Figure 2. It should be noted that vectorized image created with above mentioned patch sizes specifies the Dataset1, Dataset-2 and Dataset-3, respectively.

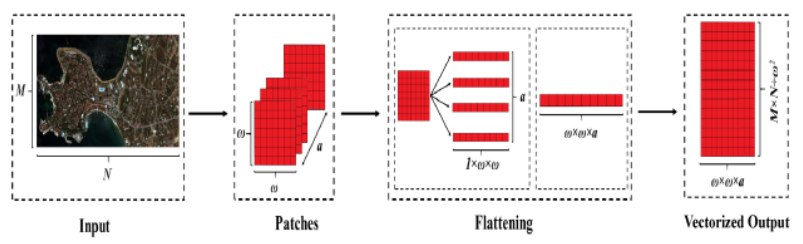

Figure 2. Creation of vectorized input from MS raster image

In the second step of patch-based classification, training and test samples were separately created from all Datasets. Training samples were formed to construct classification models with XGBoost and CatBoost classifiers and these models applied to test samples to determinate suitable user-defined parameters of algorithms. It should be noted that during the determination of training samples, the purest patches indicating highest spectral similarity between the pixels within the patch covering approximately the same location were selected to obtain more objective results. Number of training and test patches utilized to produce LULC maps using WV-2 following patch-wise classification process were given in Table 1 .

\begin{tabular}{|ccc|}
\hline Datasets & $\begin{array}{c}\text { Number of Training } \\
\text { Patches }\end{array}$ & $\begin{array}{c}\text { Number of Test } \\
\text { Patches }\end{array}$ \\
\hline Dataset- 1 & Per class: 40 & Per class: 20 \\
$(11 \times 11)$ & Total: 320 & Total: 160 \\
\hline Dataset- 2 & Per class: 120 & Per class: 60 \\
$(7 \times 7)$ & Total: 960 & Total: 480 \\
\hline Dataset-3 & Per class: 360 & Per class: 180 \\
$(3 \times 3)$ & Total: 2880 & Total: 1440 \\
\hline
\end{tabular}

Table 1: Distribution of train test patches for each Dataset

To compare the accuracy of thematic maps using different classification techniques, WV-2 image was also classified by applying pixel-based technique using XGBoost and CatBoost algorithms. To perform pixel-based classification process, 3,240 pixels for each class (i.e., total of 25,920 pixels) were chosen as training samples and test dataset consisted of totally 12,960 pixels (i.e., 1,680 pixels for each LULC classes). To construct 
the most accurate classification models using training data, optimal parameters of algorithms were determined using test dataset. After this step, thematic maps produced by both classifiers separately.

\subsection{Classification Algorithms}

\subsubsection{Extreme gradient boosting (XGBoost)}

XGBoost, one of the gradient boosting algorithm used in many remote sensing classification applications due to its robust and efficient performance (Georganos et al., 2018; Ghatkar et al., 2019; Zhang et al., 2020), was developed by Chen and Guestrin (2016). The main principle behind XGBoost is to recursively construct decision trees and to focus on correcting errors of misclassified samples of the training dataset in the next iteration instead of the correctly classified sample at previous iteration (Sahin, 2020). To achieve this purpose and to obtain the most accurate model, it applies the loss function to weak learners of previous iteration and in order to minimize overfitting problem, it utilizes regularization parameter values (Hamedianfar et al., 2020; Ustuner et al., 2020). To employ XGBoost algorithm, "xgboost" package available in $\mathrm{R}$ software was used and optimal values for user-defined parameters namely, "eta", "gamma", "min_child_weight", "subsample", "colsample_bytree", "max_depth" and "nround" were determined with grid search algorithm. Determined optimal parameter values of XGBoost were given in Table 2. It should be noted that parameter values of patch-based classification were specified for Dataset-3 and same values were employed to Dataset-1 and Dataset-2, respectively.

\begin{tabular}{|ccc|}
\hline Parameters & $\begin{array}{c}\text { Patch-Based } \\
\text { Classification }\end{array}$ & $\begin{array}{c}\text { Pixel-Based } \\
\text { Classification }\end{array}$ \\
\hline eta & 0.3 & 0.1 \\
gamma & 0 & 0 \\
min_child_weight & 0.6 & 1 \\
subsample & 0.8 & 0.8 \\
colsample_bytree & 1 & 0.4 \\
max_depth & 4 & 4 \\
nround & 200 & 300 \\
\hline
\end{tabular}

Table 2. Parameter values of XGBoost

\subsubsection{Categorical boosting (CatBoost)}

CatBoost, relatively new tree-based ensemble learning algorithms developed by Yandex (2018) for handling categorical and numerical features, have been utilized in many studies including classification of remote sensing datasets (Pham et al., 2020; Rumora et al., 2020; Bui et al., 2021; Luo et al., 2021). In the implementation of CatBoost algorithms, there are two main processing steps for training phase. First, training samples were split into subsamples using random permutation technique to robust the classification model and the labels of samples are converted into numerical values. Second, numerical values are transformed into categorical features. "catboost" package available in the $\mathrm{R}$ software was used to implement of CatBoost classifier.

\begin{tabular}{|ccc|}
\hline Parameters & $\begin{array}{c}\text { Patch-Based } \\
\text { Classification }\end{array}$ & $\begin{array}{c}\text { Pixel-Based } \\
\text { Classification }\end{array}$ \\
\hline depth & 4 & 4 \\
learning_rate & 0.3 & 0.3 \\
iteration & 400 & 300 \\
12_leaf_reg & 0.7 & 0.2 \\
rms & 0.95 & 0.95 \\
border_count & 128 & 128 \\
\hline
\end{tabular}

Table 3. Parameter values of CatBoost
The "depth", "learning_rate", "12_leaf_reg", "rms" and "border_count" parameters were tuned using grid search algorithm and the estimated parameter values for patch-based and pixel-based classifications were presented in Table 3. Note that the parameter values determined for the Dataset- 3 were kept constant within the classification models created for the other datasets.

\subsection{Accuracy Assessment}

In this study, various classification techniques were used to produce thematic maps representing 8 LULC classes using the WV-2 image. In other words, spectral values of images extracted to vectors with different size and these vectors were classified by XGBoost and CatBoost. In addition, pixel-based image classification process was also conducted and two thematic maps were generated by classification algorithms. To evaluate the accuracy of LULC maps and to compare classification performances of both classifiers, 1,000 pixels for each class (i.e., totally 8,000 pixels) were selected as validation samples. Overall accuracy (OA) and Kappa coefficient values were estimated using validation samples to conduct accuracy assessment. In addition, F-score values based on harmonic means of user's accuracy and producer's accuracy value of each LULC class were also calculated to compare class-level accuracies.

\section{RESULTS}

In this study, thematic maps of the study area were produced by XGBoost and CatBoost ensemble methods using two different image classification techniques. To clearly examine the results of classified WV-2 image, results were presented under two subsections for each classification techniques.

\subsection{Results of Patch-Based Classification}

As stated above, one of the main purposes of this study is to evaluate the effect of varying window (i.e., patch) sizes on classification accuracy. For this purpose, pixel values of WV-2 image in the $11 \times 11$ (i.e., Dataset-1), $7 \times 7$ (i.e., Dataset-2) and $3 \times 3$ (i.e., Dataset-3) window sizes were aggregated in the vectors and created vectors were utilized as new classification inputs. Classification models were formed by both classifiers using aforementioned parameters and thematic maps were produced.

\begin{tabular}{|c|cccccc|}
\hline \multirow{2}{*}{ LULC } & \multicolumn{6}{|c|}{ F-score Values } \\
\cline { 2 - 7 } Classes & \multicolumn{2}{|c|}{ Dataset-1 } & \multicolumn{2}{c|}{ Dataset-2 } \\
\cline { 2 - 7 } & XGB & CB & XGB & CB & XGB & CB \\
\hline Forest & 66.17 & 71.44 & 83.01 & 85.80 & 92.81 & 93.33 \\
Gray S. & 49.53 & 51.35 & 72.35 & 72.23 & 85.49 & 87.34 \\
Pasture & 69.92 & 66.49 & 81.44 & 81.65 & 90.83 & 92.25 \\
Shadow & 77.11 & 74.96 & 82.72 & 84.80 & 93.82 & 93.37 \\
Soil & 70.70 & 71.19 & 81.64 & 83.21 & 91.04 & 90.01 \\
Tile R. & 72.96 & 74.29 & 84.01 & 83.67 & 94.85 & 94.16 \\
Water & 71.03 & 75.85 & 82.76 & 86.68 & 92.44 & 94.35 \\
White S. & 67.43 & 62.87 & 87.19 & 82.96 & 98.07 & 97.76 \\
\hline OA (\%) & 67.83 & 68.05 & 81.62 & 82.40 & 92.31 & 92.75 \\
\hline Kappa & 0.63 & 0.64 & 0.79 & 0.80 & 0.91 & 0.92 \\
\hline \multicolumn{7}{|c}{ Table 4. Accuracy results of maps produced by patch-based } \\
\multicolumn{7}{c}{ classification } \\
\end{tabular}

Accuracy assessment results of each thematic map produced by XGBoost and CatBoost were presented in Table 4. By the analysing of table, it was seen that there was an inverse relationship between patch-size considered and estimated classification accuracies. In other words, the highest $\mathrm{OA}$ values 
(i.e., \%92.31 with Kappa coefficient value of 0.91 by XGBoost and \%92.75 with Kappa coefficient value of 0.92 by CatBoost) were observed dividing WV-2 image to $3 \times 3$ patches (Dataset3 ), whereas the lowest OA values were estimated as $67.83 \%$ (Kappa coefficient value of 0.63) and 68.05\% (Kappa coefficient value of 0.64 ) by XGBoost and CatBoost, respectively with the use of $11 \times 11$ patch size. In addition, the surface objects in the study area were assigned to LULC classes using Dataset-2 with an OA value of \%81.62 (Kappa coefficient value of 0.79 ) and \%82.4 (Kappa coefficient value of 0.80 ) by XGBoost and CatBoost classifiers, respectively. Classification outputs showed that use of smaller patches for producing thematic maps enhanced the OA values up to \%10 and \%24 compared to classification of surface objects with Dataset-1. Furthermore, it was found that the CatBoost classifier outperformed the XGBoost method with respect to the estimated OA values in all cases.

In this study, F-score measure indicating the individual classaccuracies were also assessed. When the results of Dataset-1 analysed, it was seen that estimated class level accuracies for both classifiers below the $78 \%$. In addition, the lowest F-score values as $\% 49.53$ as $\% 51.35$ calculated for gray surface class by, whereas shadow and water were classified by XGBoost and CatBoost with the highest class-level accuracy $77.11 \%$ and $75.85 \%$, respectively. When the F-score results calculated utilizing Dataset-2 were evaluated, about all objects were attached to relevant classes by both algorithms with over $\% 81$ accuracy except gray surface class. On the other hand, the improvement in individual class-level of forest, shadow and soil reached to about \%2 using CatBoost compared to XGBoost. However, classifying white surface using XGBoost resulted in approximately $\% 5$ better accuracy than result of Catboost. The results of class-based measurements of Dataset- 3 showed that XGBoost and CatBoost determined the boundaries of nearly all classes with over $\% 90$ F-score values. On the other hand, when white surface was classified with highest value by both algorithms, the worst F-score values were estimated for gray surface class which was significantly lower than results of other classes as nearly $\% 4$. When the classification performance of the algorithms was evaluated for all data sets, CatBoost was produced better results for forest and water class. However, XGBoost was outperformed to CatBoost in identifying boundaries of white surface class in all cases.

Visual results produced by XGBoost and CatBoost using Dataset-1, Dataset-2 and Dataset-3 were presented separately in Figure 3. As could be seen from figure, smoother maps were obtained when WV-2 image was classified utilizing smaller patch size (i.e., 3x3). In other words, results of Dataset-1 generated by both algorithms had the nosiest look, whereas maps of Dataset- 3 were smoothest. On the other hand, visual results indicated that boundaries of LULC classes were more accurately drawn using Dataset-3, yet classes covering large areas such as water were mapped more clearly by the use Dataset-3. In addition, misclassification errors were determined between shadow and water classes. When CatBoost tended to classify the water class as shadow, XGBoost was found to have superior classification performance in identifying these classes.

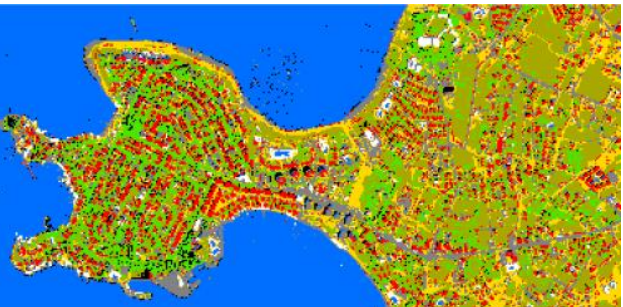

(a)

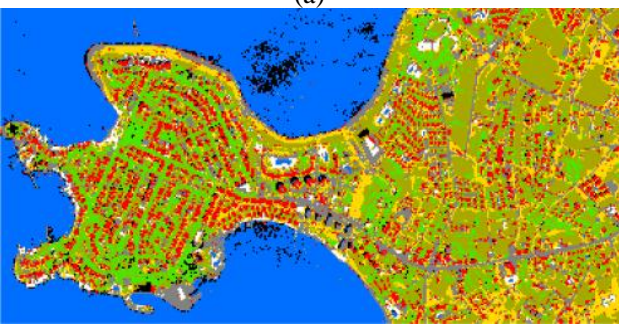

(b)

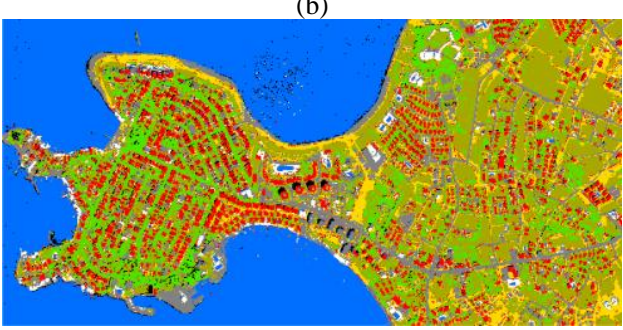

(c)

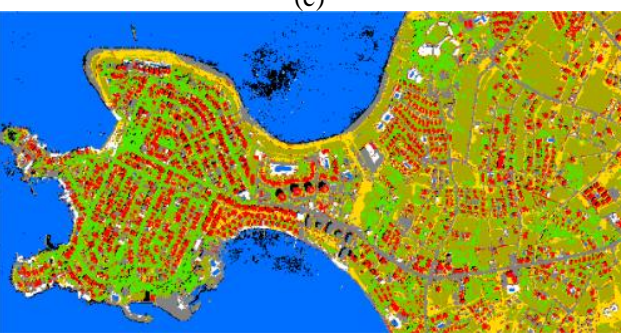

(d)

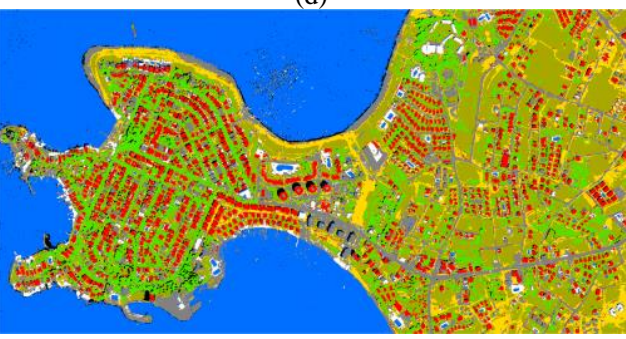

(e)

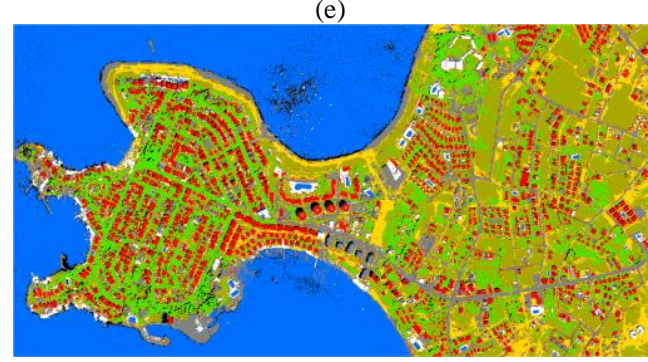

(f)
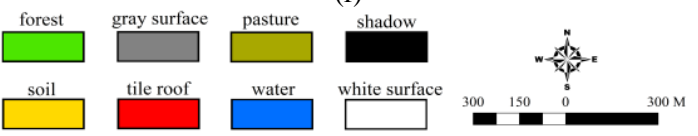

Figure 3. Thematic maps produced using Dataset-1 by (a) XGBoost, (b) CatBoost; using Dataset-2 by (c) XGBoost, (d) CatBoost; using Dataset-3 by (e) XGBoost, (f) CatBoost 


\subsection{Results of Pixel-Based Classification}

In order to compare the performance of patch-based classification results of XGBoost and CatBoost algorithms, the WV-2 imagery was also classified using traditional pixel-based classification technique. Classification models were constructed using determined user-defined parameters applied whole dataset and thematic maps were produced. Thematic map accuracies were calculated using confusion matrix-derived measures including OA, Kappa coefficient and F-Score values. Table 5 shows the pixel-based results of LULC maps yielded by XGBoost and CatBoost. Moreover, it was found that maps with similar OA values were generated by both classification algorithms. In other words, when the surface objects of study area depicting eight LULC classes were mapped of \%94.65 OA value (Kappa coefficient of 0.94) via CatBoost, XGBoost classified these objects with OA of \%94.59 (Kappa coefficient of 0.94 ), with a $\% 0.06$ difference. These classification results showed that pixel-based image analysis method produced more accurate and efficient maps by both robust classifiers compared to patch-based classification process for this study.

\begin{tabular}{|c|cc|}
\hline \multirow{2}{*}{ LULC } & \multicolumn{2}{|c|}{ F-score Values (\%) } \\
\cline { 2 - 3 } Classes & XGB & CB \\
\hline Forest & 94.94 & 94.58 \\
Gray Surface & 90.83 & 91.11 \\
Pasture & 93.23 & 93.21 \\
Shadow & 96.47 & 96.47 \\
Soil & 91.97 & 91.40 \\
Tile Roof & 93.76 & 93.96 \\
Water & 96.41 & 96.98 \\
White Surface & 99.30 & 99.65 \\
\hline OA (\%) & 94.59 & 94.65 \\
\hline Kappa & 0.94 & 0.94 \\
\hline
\end{tabular}

Table 5. Accuracy results of maps produced by pixel-based classification

F-sore values were computed for comparing class-based accuracies generated by XGBoost and CatBoost and measurements were given in Table 5 . When the table was analysed, mapping of each LULC class resulted in with accuracy of over $90 \%$ using pixel-based classification procedure. On the other hand, when the highest F-score values calculated by classification algorithms were observed for white surface class over $99 \%$ accuracy, surface and soil classes were classified with worst accuracies of about $91 \%$. In addition, XGBoost showed superior classification performance for forest, pasture, and soil, whereas gray surface, tile roof, water and white surface classes were more accurately classified by CatBoost. Furthermore, it was seen that boundaries of shadow class were determined with same accuracy level (i.e., 96.47\%) by classifiers.

Thematic maps produced by pixel-based XGBoost and CatBoost classifier were given in Figure 4. As can be seen from the figure, both classifiers mapped the LULC classes approximately the same accuracy using pixel-based image analysis. In addition, main classification errors were determined between gray surface and water. The reason for this error is thought to be caused by gravels under water in shallow areas. When the maps with the highest OA value produced as patchbased (i.e., Dataset-3) were compared with the images produced as pixel-based, it was found that the classification error between water and shadow classes was eliminated by using pixel-based image analysis technique.

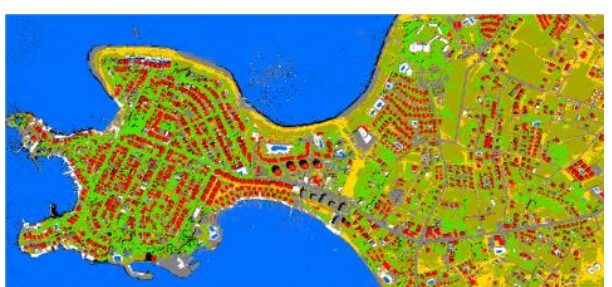

(a)

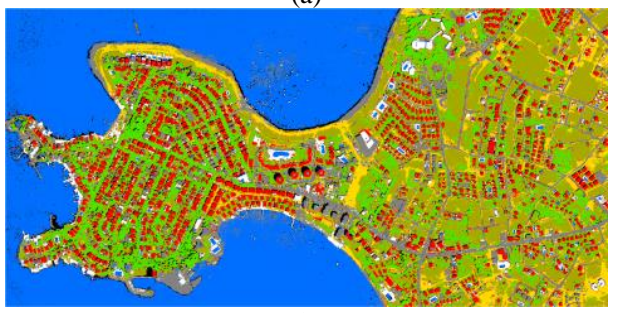

(b)

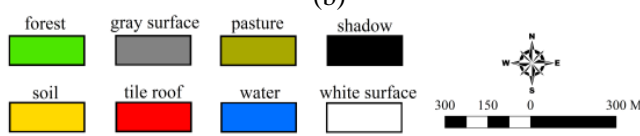

Figure 4. Thematic maps produced using pixel-based image analysis technique by (a) XGBoost, (b) CatBoost

\section{CONCLUSIONS}

In this study, the accuracies of thematic maps produced by two different classification techniques were analysed. For this purpose, high spatial resolution WV-2 image was classified applying patch-based classification process using various window sizes and pixel-based classification process. Robust ensemble learning methods, XGBoost and CatBoost, were used to implementation of classification task. LULC maps of study area were generated by these classifiers using mentioned classification techniques and results of maps were evaluated with OA, Kappa coefficient and F-score measurements.

Some important findings were obtained from the classification results of this study. Firstly, in the patch-based classification, the most accurate thematic maps with over $\% 92$ OA values were produced with the use of Dataset- 3 by both classifiers. On the other hand, employing classification models using Dataset-1 and Dataset-2 decreased the OA values of maps up to about $\% 68$ and $\% 82$ respectively. These results verified that the use of smaller window size in the classification of raster image with patch-based procedure improved the classification accuracy. Secondly, thematic maps yielded by pixel-based image analysis technique produced more accurate results compared to maps produced extracting pixel values of image to vectors with different sizes and improvements in map accuracies reached to about $\% 2, \% 12$ and $\% 27$, respectively. Moreover, the result showed that pixel-based classification technique can be produce more accurate results using ensemble learning algorithms. Thirdly, when the thematic maps produced by patch-based (patch size of $3 \times 3$ ) and pixel-based analyzed separately, patchbased approach showed superior performance in the classification of the interior areas of objects. However, it was insufficient of assigning object edges to the correct class label. On the other hand, pixel-based classification approach outperformed to patch-based classification in the determination of the class boundaries clearly. This finding showed that while better results can be obtained with pixel-based classification in the objects with sharp boundaries, maps containing lower level noise (i.e., salt-and-pepper noise) can be produced with patchbased classification. Finally, CatBoost yielded better classification results than XGBoost method in all cases. In other 
words, although both algorithms produced thematic maps with similar accuracies in patch-based classification and pixel-based classification process, improvement in accuracies increased about $\% 0.5$ in the patch-based classification and \%0.06 in the pixel-based classification by using CatBoost comparing to XGBoost. The superiority of CatBoost may be related the training approach that convert categorical features to numeric. More studies should conduct to compare the performance of pixel-based and patch-based approach in the classification of the datasets including different object types. This study was carried out in a small area. High resolution LULC maps to be produced for larger areas will contribute more to the management and planning of smart cities.

\section{REFERENCES}

Bui, Q.-T., Chou, T.-Y., Hoang, T.-V., Fang, Y.-M., Mu, C.-Y., Huang, P.-H., Pham, V.-D., Nguyen, Q.-H., Anh, D. T. N., Pham, V.-M., Meadows, M.E., 2021. Gradient Boosting Machine and Object-Based CNN for Land Cover Classification. Remote Sensing, 2709. https://doi.org/10.3390/rs13142709

Chen, T., Guestrin, C., 2016. XGBoost. Proceedings of the 22nd ACM SIGKDD International Conference on Knowledge Discovery and Data Mining, 13-17-Augu, 785-794. https://doi.org/10.1145/2939672.2939785

Colkesen, I., Ertekin, O.H., 2020. Performance Analysis of Advanced Decision Forest Algorithms in Hyperspectral Image Classification. Photogrammetric Engineering \& Remote Sensing, 86(9), 571-580. https://doi.org/10.14358/PERS.86.9.571

Colkesen, I., Kavzoglu, T., 2017. The use of logistic model tree (LMT) for pixel- and object-based classifications using highresolution WorldView-2 imagery. Geocarto International, 32(1), 71-86. https://doi.org/10.1080/10106049.2015.1128486

Dingle Robertson, L., King, D.J., 2011. Comparison of pixeland object-based classification in land cover change mapping. International Journal of Remote Sensing, 32(6), 1505-1529. https://doi.org/10.1080/01431160903571791

Dumitru, C.O., Schwarz, G., Cui, S., Datcu, M., 2016. Improved image classification by proper patch size selection: TerraSAR-X vs. Sentinel-1A. 2016 International Conference on Systems, Signals and Image Processing (IWSSIP), 2016-June, 1-4. https://doi.org/10.1109/IWSSIP.2016.7502739

Gašparović, M., Dobrinić, D., 2020. Comparative Assessment of Machine Learning Methods for Urban Vegetation Mapping Using Multitemporal Sentinel-1 Imagery. Remote Sensing, 12(12), 1952. https://doi.org/10.3390/rs12121952

Georganos, S., Grippa, T., Vanhuysse, S., Lennert, M., Shimoni, M., Wolff, E., 2018. Very High Resolution ObjectBased Land Use-Land Cover Urban Classification Using Extreme Gradient Boosting. IEEE Geoscience and Remote Sensing Letters, 15(4), 607-611. https://doi.org/10.1109/LGRS.2018.2803259

Ghatkar, J.G., Singh, R.K., Shanmugam, P., 2019. Classification of algal bloom species from remote sensing data using an extreme gradient boosted decision tree model. International Journal of Remote Sensing, 40(24), 9412-9438. https://doi.org/10.1080/01431161.2019.1633696
Hamedianfar, A., Gibril, M.B.A., Hosseinpoor, M., Pellikka, P.K.E., 2020. Synergistic use of particle swarm optimization, artificial neural network, and extreme gradient boosting algorithms for urban LULC mapping from WorldView-3 images. Geocarto International, 0(0), 1-19. https://doi.org/10.1080/10106049.2020.1737974

Immitzer, M., Atzberger, C., Koukal, T., 2012. Tree Species Classification with Random Forest Using Very High Spatial Resolution 8-Band WorldView-2 Satellite Data. Remote Sensing, 4(9), 2661-2693. https://doi.org/10.3390/rs4092661

Islam, M.D., Islam, K.S., Mia, M., 2020. An XGBoost Based Approach for Urban Land Use and Land Cover Change Modelling. Authorea Preprints, 1-13. https://doi.org/10.22541/au.159646139.97656606

Lebedev, A.V., Zavarzin, V.V., Gemonov, A.V., 2020. Vegetation Cover Change in Kologrivsky Forest Nature Reserve Detected using Landsat Satellite Image Analysis. IOP Conference Series: Earth and Environmental Science, 507(1), 012016. https://doi.org/10.1088/1755-1315/507/1/012016

Lechner, A.M., Stein, A., Jones, S.D., Ferwerda, J.G., 2009. Remote sensing of small and linear features: Quantifying the effects of patch size and length, grid position and detectability on land cover mapping. Remote Sensing of Environment, 113(10), 2194-2204. https://doi.org/10.1016/j.rse.2009.06.002

Lizarazo, I., Elsner, P., 2009. Fuzzy segmentation for object-based image classification. International Journal of Remote Sensing, 30(6), 1643-1649. https://doi.org/10.1080/01431160802460062

Luo, J., Ma, X., Chu, Q., Xie, M., Cao, Y., 2021. Characterizing the Up-To-Date Land-Use and Land-Cover Change in Xiong'an New Area from 2017 to 2020 Using the Multi-Temporal Sentinel-2 Images on Google Earth Engine. ISPRS International Journal of Geo-Information, 10(7), 464. https://doi.org/10.3390/ijgi10070464

Luo, M., Wang, Y., Xie, Y., Zhou, L., Qiao, J., Qiu, S., Sun, Y., 2021. Combination of Feature Selection and CatBoost for Prediction: The First Application to the Estimation of Aboveground Biomass. Forests, 12(2), 216. https://doi.org/10.3390/f12020216

Omer, G., Mutanga, O., Abdel-Rahman, E.M., Adam, E. 2015. Exploring the utility of the additional WorldView-2 bands and support vector machines in mapping land use/land cover in a fragmented ecosystem, South Africa. South African Journal of Geomatics, 4(4), 414. https://doi.org/10.4314/sajg.v4i4.5

Ozturk, M.Y., Colkesen, I., 2021. Investigation of the effects of vegetation indices derived from UAV-based RGB imagery on land cover classification accuracy Intercontinental Geoinformation Days Investigation of the effects of vegetation indices derived from UAV-based RGB imagery on land. 2nd Intercontinental Geoinformation Days (IGD), May, 1-5.

Pal, M., Himanshu, R., Teja, B.C., 2020. Patch Based Classification of Remote Sensing Data: A Comparison of 2DCNN, SVM and NN Classifiers. ArXiv Preprint ArXiv:2006.11767, Dl.

Pham, T.D., Yokoya, N., Nguyen, T.T.T., Le, N.N., Ha, N.T., Xia, J., Takeuchi, W., Pham, T.D., 2020. Improvement of 
Mangrove Soil Carbon Stocks Estimation in North Vietnam Using Sentinel-2 Data and Machine Learning Approach. GIScience \& Remote Sensing, 1-20. https://doi.org/10.1080/15481603.2020.1857623

Rumora, L., Miler, M., Medak, D., 2020. Impact of Various Atmospheric Corrections on Sentinel-2 Land Cover Classification Accuracy Using Machine Learning Classifiers. ISPRS International Journal of Geo-Information, 9(4), 277. https://doi.org/10.3390/ijgi9040277

Sahin, E.K., 2020. Comparative analysis of gradient boosting algorithms for landslide susceptibility mapping. Geocarto International, $0(0), \quad 1-25$. https://doi.org/10.1080/10106049.2020.1831623

Samat, A., Li, E., Du, P., Liu, S., Miao, Z., Zhang, W., 2020. CatBoost for RS Image Classification With Pseudo Label Support From Neighbor Patches-Based Clustering. IEEE Geoscience and Remote Sensing Letters, 1-5. https://doi.org/10.1109/LGRS.2020.3038771

Sharma, A., Liu, X., Yang, X., Shi, D. 2017. A patch-based convolutional neural network for remote sensing image classification. Neural Networks, 95, 19-28. https://doi.org/10.1016/j.neunet.2017.07.017

Ustuner, M., Abdikan, S., Bilgin, G., Balik Sanli, F., (2020). Hafif Gradyan Artırma Makineleri ile Tarımsal Ürünlerin Sınıflandırılması. Türk Uzaktan Algılama ve CBS Dergisi, 1(2), 97-105.

Xu, L., Zhang, H., Wang, C., Zhang, B., Liu, M., 2018. Crop Classification Based on Temporal Information Using Sentinel-1 SAR Time-Series Data. Remote Sensing, 11(1), 53. https://doi.org/10.3390/rs11010053

Yang, R., Hu, Z., Liu, Y., Xu, Z., 2020. A Novel Polarimetric SAR Classification Method Integrating Pixel-Based and PatchBased Classification. IEEE Geoscience and Remote Sensing Letters, 17(3), 431-435. https://doi.org/10.1109/LGRS.2019.2923403

Zhang, W., Liu, H., Wu, W., Zhan, L., Wei, J., 2020. Mapping rice paddy based on machine learning with sentinel-2 multitemporal data: Model comparison and transferability. Remote Sensing, 12(10). https://doi.org/10.3390/rs12101620

Zheng, H., Du, P., Chen, J., Xia, J., Li, E., Xu, Z., Li, X., Yokoya, N., (2017). Performance Evaluation of Downscaling Sentinel-2 Imagery for Land Use and Land Cover Classification by Spectral-Spatial Features. Remote Sensing, 9(12), 1274. https://doi.org/10.3390/rs9121274

Zhu, X.X., Tuia, D., Mou, L., Xia, G.-S., Zhang, L., Xu, F., Fraundorfer, F., 2017. Deep Learning in Remote Sensing: A Comprehensive Review and List of Resources. IEEE Geoscience and Remote Sensing Magazine, 5(4), 8-36. https://doi.org/10.1109/MGRS.2017.2762307 